à un état final (correspoudant ì un nouvel élal de transmission) :

$$
\mu, \mu x, x^{2}, \ldots, \text { i } x^{n}, \ldots, \mu x^{N} \text {. }
$$

Au commencement de la transition, le levier de mesure occupe la position correspondant ì une déformation connue $T_{0}$ de la circonférence du tube. Désignons par $M_{n}$ et $T_{n}$ les valeurs que l'on observe à la fin du stade $n$ et, par $m_{n}$ et $I_{n}$ les accroissements subis par ces grandeurs au cours du stade n. On a donc, par définition,
(6)

$$
m_{\mathrm{n}}=\mathrm{M}_{\mathrm{n}}-\mathrm{M}_{\mathrm{n}-1}
$$$$
\text { et } t_{n}=T_{n}-T_{n-1}
$$

A la fin du stade $(n-1)$, les segments 0 à $(n-1)$ sont en état de transmission; au cours du stade $n$, le tube subit unc déformation $t_{n}$ telle que l'accroissement de tension

$$
\mathrm{a}_{\mathrm{n}}=\frac{\mathrm{t}_{\mathrm{n}}}{\rho \mathrm{N}_{1} \mathrm{bF}}
$$

qui en résulte pour le segment $n$, amène cellui-ci, à son tour, à l'état de transmission ; $m_{n}$, le déplacement du levier correspondant à la déformation $t_{n}$, mesure la déformation de l'arc de circonférence-guide relatif aux segments 0 à $(\mathbf{n}-1)$, diminuée de la déformation élastique du ruban qui doit passer au cours du slade $n$, des tensions relatives :

$$
j_{n-1}, j_{n-1} x, j_{n-1} x^{2}, \ldots, j_{n-1} x^{n-1} \text {, }
$$

mux tensions relatives :

$$
\mu_{11}, \quad \mu_{11} x, \quad \mu_{11} x^{2}, \quad \ldots, \quad \mu_{n 1} x^{n-1} .
$$

On peul donc ócrire :

$\mathrm{m}_{n}=\mathrm{bF} \mathrm{a}_{\mathrm{n}}\left(\mathrm{n}+x_{0}-1\right)-\mathrm{bF}\left(\mu_{n}-\mu_{n 1}\right)\left(X_{n}+\beta_{0}-1\right)$. d'où, en remarquant que,

avec la notalion :

$$
\begin{gathered}
m_{n}=\frac{\left(b F\left(n+\alpha_{0}-1\right)\right.}{a_{n}}=\frac{\left(M_{n-1}-M_{n-1}\right)=}{1+\rho b F\left(X_{n}+\beta_{n}-1\right.} \text { ou } \frac{m_{n}}{a_{n}}=g_{n}(x),
\end{gathered}
$$

$$
g_{n}(x)=\frac{b F\left(n+x_{0}-1\right)}{1+p b F\left(X_{n}+\beta_{0}-1\right.}
$$

Le rapport d'enregisliment, $\mathrm{m}_{\mathrm{n}} / \mathrm{l}_{\mathrm{n}}$, dun stade donne, ainsi que le rapport $\mathrm{m}_{\mathrm{n}} / \mathrm{a}_{\mathrm{n}}$, est indépendant de l'élat de lension du ruban ; il ne dépend que du numéro d'orde du slade et du sens dans lequel se fait la déformalion, soit de $\mathrm{g}_{\mathrm{n}}(\mathrm{u})$ pour l'extension et de $\mathrm{g}_{\mathrm{n}}(\mathrm{r})$ pour la contraction.

Le problème que nous arons à résoudro se limite donc ì la détermination de l'une quelconque des trois quantités, $a_{n}$, $m_{n}$ ou $t_{n}$, les deux autres s'en déduisant par les formules (7) et (9), à moins qu’il soit possible d'établir des formules reliant directement $\left(M_{n}-M_{0}\right)$ à $\left(T_{n}-\right)$, sans être obligé de passer par les valeurs de stade.

\title{
La Houille Blanche et les Mines métalliques
}

\author{
par V. Gharrin
}

Avant d'entreprendre l'exploitation de tous gisements métallifères, deux points essentiels sont à étudier très attentivement en dehors de la richesse des filons : celui de la force motrice et celui de l'eau. Faute de l'avoir fail ou pour l'avoir fait d'une façon trop superficielle, de nombreuses entreprises ont eu, dans la suite, des difficultés sérieuses, qui, parfois même, les ont menées à l'échec.

Ces deux questions se confondent souvent, au point de n'en faire qu'une seule.

\section{FORCE MOTRICE}

Sauf des cas privilégiés, qui le plus sourent ne sont que temporaires. où l'exploitation de la mine se fait à flanc de coteau avec écoulement naturel des eaux, l'extraction des produits et l'exhausse exigent une force motrice importante, surtout que la plupart des filons métalliques sont fortement aquifères, amenant ainsi de gros volumes d'eau à pomper.

La perforation mécanique est indispensable pour l'abatage des minerais; mais ce sont, en général, les appareils de préparation de ces minerais pour les rendre marchands qui sont les plus gros consommateurs d'énergie.

Comme exemple, nous allons citer l'atelier de préparation mécanique des mines des Bormetles (Var) qui, pendant ilongtemps ful considéné comme un modèle du genre. Le minerai qui lui était soumis était un minerai plombo-zincifère et il s'agissait de séparer ces deux éléments, tout en les enrichissant. L'atelier pouvait débiter 220 tonnes de minerai brut par 24 heures, soil près de 9 tonnes à l'heure. Pour la marche de tous les appareils, il fallait une force de 100 chevanx et une machine à vapeur de 110 chevaux avait élé installée. Les deux tiers de cette force allaient aux concasseurs et aux pompes; ces dernières avaient à fournir aux divers appareils (concasseurs, broycurs, élévateurs, classeurs, trommels, cribles, tables) 3.230 litres d'eau à la minute.

On voit par là que dans le prix de revient du minerai marchand, la force motrice entre pour une part importante.

Le plus souvent les mines métalliques sont situées loin des sources de charbon et les tramports en rendent les prix complètement prohibitifs ; il est vrai qu'aujourd'hui d'autres moyens s'offrent au mineur ; le courant électrique sillonne à peu près tout le territoire et les moteurs à essence ou ì mazout peuvent se transporter n'importe où. Mais, dans ces deux cas, il y a encore une question de coût à faire intervenir. Le cheval vapeur produit par l'essence est très cher ; plus abordable est celui des secteurs électriques, mais pour ces derniers, des frais accessoires se greffent toujours : ligne de raccordement, poste transformateur, etc., et, de plus, la mine devient tribulaire d'un producteur qui lui cst étranger.

Nous avons vu, vers 1925, plusieurs carrières souterraines de bauxite de la région de Brignoles complètement envahies par les eaux, à la suite de la destruction d'une partie de la ligue à haute-tension Entraigues-St-Maximin, par des incendies de forêts.

Les mines mélalliques sont situées généralement en pays de montagnes et, toutes les fois qu'elles pourront utiliser la houille blanche locale, ce sera pour elles un aloul considérable. Il faudra tout de même, pour certains cas, avoir encore recours au charbon; pour los fours à cuve et les fours à calcincr qu'exige la calamine, par exemple, mais celte consommation relativement faible n'aura rien de comparable à colle nécessaire au fonctionnement des ateliers.

Les mines des Malines, dans le Gard voul nous servir a illustrer cette utilisation hydraulique. Cette entreprise dont la célébrité dura plusieurs lustres, est née en 1885 ; elle avail pour objet des minerais de zinc et de plomb argentifère ; ses travaux ont complètement cessé en 1933. Elle eut une prospérité inouïe jusqu'à la guerre, puisqu'on considéra qu'elle rapporta ̀̀ ses heureux propriétaires plus de 150 millions de francs, avec un capital initial de 136.000 franos. Ce résultat prodigieux et peut-être unique au monde, fut dû, c'est entendu, à des amas blendeux et calaminaires d'une richesse exceptionnelle et à des conditions extrêmement faciles d'exploitation - tous les travaux ont été faits à flanc de monta- 
gne -, mais l'emploi de la force motrice produite par la houille blanche n'y fut pas totalement étranger non plus.

Non loin des travaux d'exploitation el à peu près à michemin de la gare P.L.M. de Ganges (Hérault), par laquelle s'expédiaient les produits, la société installa ses ateliers de préparation mécanique, complètement en bordure de la rivière la Vis, affluent de l'Hérault. Cette rivière, à régime quelque peu torrentiel, n'a jamais d'étiage très bas, car les terrains calcaires fortement crevassés qui forment son bassin constituent de véritables réscrvoirs.

Trois chutes furent aménagées (fig. 1) avec bassin d'alimentation, de chacune 40 chevaux environ, et, à part exception pour les quelques années oì l'extraction dépassa la moyenne el où l'on ful obligé de demander le supplément de force à des machines thermiques, elles suffirent, en temps normal, pour assurer tous les services.

Une de ces chutes, celle dite de la Papeleric, entrainait directement l'atclier d'enrichissement des calamines; une autre, la plus en' aval, alimentait par l'intermédiaire du courant électrique, l'atelier de traitement des blendes ; la troisième, dite chute de la Meluse, assurait tous les services accessoires, y compris l'atelier des blendes barytiques traitées au procédé Murex (précurseur de la flottation).

On peut évidemment reprocher à cette conception la dispersion, ćtant entendu qu'une complète centralisation aurait l'avons dit au début de celte note, c'est une étude préalable approfondie qu'il faut dans chaque cas, avant de ne rien entreprendre.

\section{$\begin{array}{llll}E & A & U\end{array}$}

L'eau qui, comme nous venons de le voir, peul résoudre économiquement la question de force motrice, est indispensable pour le traitement des minerais. De plus en plus, on a tendance à concasser, broyer et cribler à l'eau ; en tous cas, on ne peut s'en passer pour les cribles el les tables.

Il est assez difficile d'évaluer la quantité d'eau qui sera nécessaire pour le traitement; elle, varie d'ailleurs avec la nature du minerai, celle de la gangue et aussi avec le nombre des éléments à séparer ; dans les minerais courants de plomb et de zinc, il faut compter de 15 à 20 mètres cubes par tonne de minerai brut.

Souvent, on alimente les laveries avec l'eau sortant des travaux souterrains, mais cette pratique n'est pas toujours recommandable car, si ces eaux sont sulfureuses - _cas très fréquent dans les mines où existe la pyrite - elles détériorent rapidement toutes les parties métalliques des appareils.

Assez souvent aussi, devant l'insuffisance d'eau, on récupère celle-ci après usage et on procède à sa décantation dans les bassins construits dans ce but, avant de la faire servir à nouveau. Les frais d'entretien de ces bassins et leur curage grèvent alors les prix de revient.

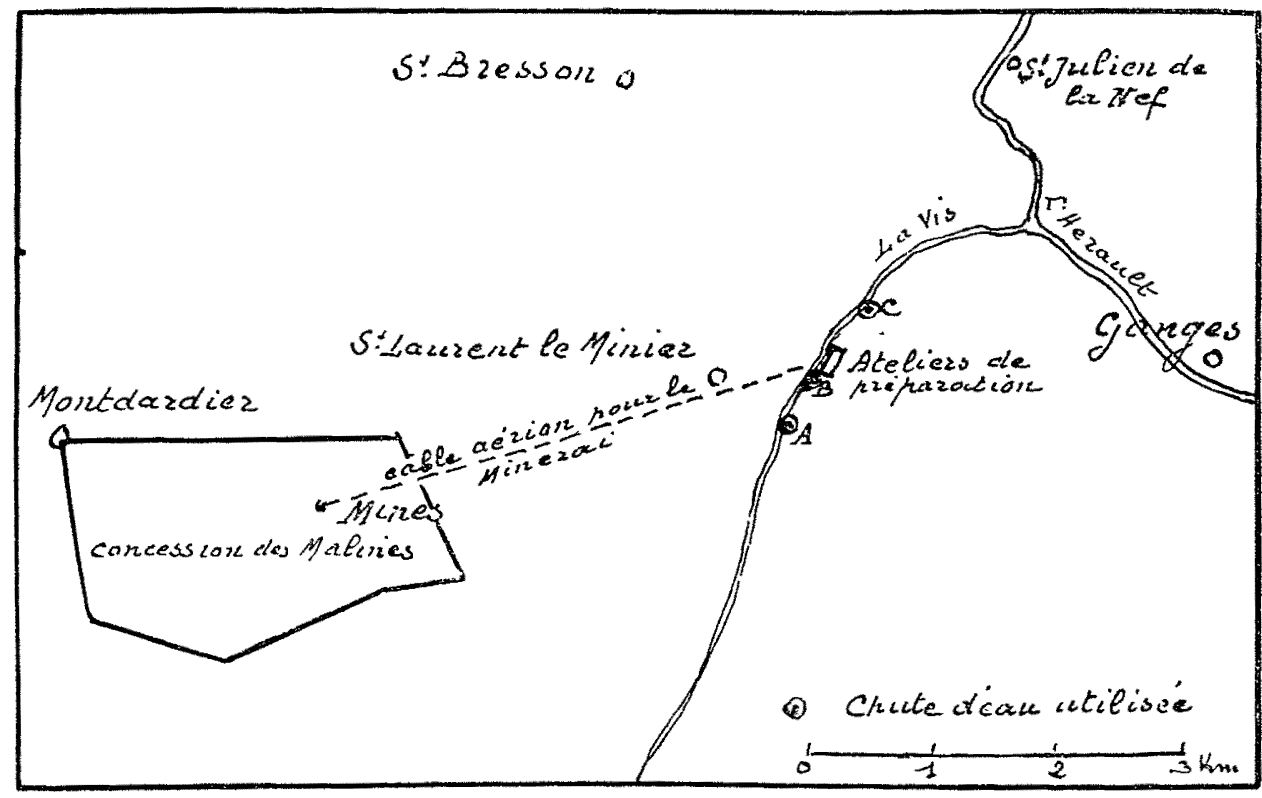

Litilisation de la horille blanche aux mines des Malines.

amené une marche encore plus économique ; néanmoins, on ne peutl que s'incliner devant les résultats qui permirent le travail jusqu'en 1932, malgré l'effondrement des cours du plomb, du zinc et de l'argent, avec des minerais cependant assez paurres, puisqu'ils ne renfermaient que 12 à $15 \%$ de métal.

Ce sera d'ailleurs l'énergie hydraulique demandée à la Vis, mais avec un plan mieux conçu, qui permettra la reprise de cette affaire dans laquelle on est certain de l'existence d'un gros tonnage de minerais à teneur relativement basse, mais qui peuvent parfaitement conduire à des amas analogues à ceux qui ont fait la fortune diu passé.

l'exemple des Malines peut servir dans de très nombreux cas ; Jes gisements métallifòres des Alpes, des Pyrénées, du Massif Central ont tous, à distance raisonnable, des cours d'eau qui peuvent leur fournir l'énergie à bon marché et souvent rendre ainsi leur exploitation possible; comme nous
L'eau abondante, on le voit, est de toute nćcessité pour le fonctionnement normal et économique de tout atelier de préparation mécanique des minerais ; on a parfois intérèt à conduire ceux-ci même à grande distance pour que cette condition ful remplie.

La mine des Deux-Jumeaux à Sumène (Gard) est le prototype de l'exploitation métallique qui manque d'cau quoique placće sur les bords de la rivière Riotord ; celle-ci d'ailleurs de régime torrentiel, est à sec les trois-quarts de l'année, par suite des infiltrations dans les graviers. Une petite installation de laverie pour traiter les blendes, quoique bien modeste, n'a jamais pu fonctionner faute d'cau pour l'alimenter.

En résumé, toute mine métallique, qui, comme celle des Malines, pourra pratiquement conduire ses minerais sur un cours d'eau qui lui fournira la force motrice et l'eau des divers services, aura un des points épineux du problème résolu. 